University of Montana

ScholarWorks at University of Montana

Physical Therapy and Rehabilitation Science

Faculty Publications

Physical Therapy and Rehabilitation Science

2004

\title{
Comparison of Surface Electromyography and Myotonometric Measurements during Voluntary Isometric Contractions
}

\author{
Charles Leonard \\ University of Montana - Missoula, charles.leonard@umontana.edu \\ Jason S. Brown \\ University of Montana - Missoula \\ Timothy R. Price \\ University of Montana - Missoula \\ Susan A. Queen \\ University of Kentucky, Lexington, $K Y$ \\ Eugene L. Mikhailenok \\ Neurogenic Technologies, Inc., Missoula, MT
}

Follow this and additional works at: https://scholarworks.umt.edu/ptrs_pubs

Part of the Rehabilitation and Therapy Commons

Let us know how access to this document benefits you.

\section{Recommended Citation}

Leonard, Charles; Brown, Jason S.; Price, Timothy R.; Queen, Susan A.; and Mikhailenok, Eugene L., "Comparison of Surface Electromyography and Myotonometric Measurements during Voluntary Isometric Contractions" (2004). Physical Therapy and Rehabilitation Science Faculty Publications. 2.

https://scholarworks.umt.edu/ptrs_pubs/2

This Article is brought to you for free and open access by the Physical Therapy and Rehabilitation Science at ScholarWorks at University of Montana. It has been accepted for inclusion in Physical Therapy and Rehabilitation Science Faculty Publications by an authorized administrator of ScholarWorks at University of Montana. For more information, please contact scholarworks@mso.umt.edu. 


\title{
Comparison of surface electromyography and myotonometric measurements during voluntary isometric contractions
}

\author{
Charles T. Leonard ${ }^{\mathrm{a}, \mathrm{b}, *}$, Jason S. Brown ${ }^{\mathrm{a}}$, Timothy R. Price ${ }^{\mathrm{a}}$, Susan A. Queen ${ }^{\mathrm{c}}$, \\ Eugene L. Mikhailenok ${ }^{\mathrm{b}}$ \\ ${ }^{a}$ Motor Control Research Laboratory, Physical Therapy Department, School of Pharmacy and Health Sciences, University of Montana, \\ Missoula, MT 59812, USA \\ ${ }^{\mathrm{b}}$ Neurogenic Technologies, Inc., 5000 Pattee Canyon Drive, Missoula, MT 59812, USA \\ c Physical Therapy Department, The University of Kentucky, 204 Charles T. Wethington Building, Lexington, KY 40536, USA
}

\begin{abstract}
Objectives: Muscle stiffness increases during muscle contraction. The purpose of this study was to determine the strength of the correlation between myotonometric measurements of muscle stiffness and surface electromyography (sEMG) measurements during various levels of voluntary isometric contractions of the biceps brachii muscle. Subjects: Eight subjects (four female; four male), with mean age of $30.6 \pm 8.23$ years, volunteered to participate in this study. Methods: Myotonometer and sEMG measurements were taken simultaneously from the right biceps brachii muscle. Data were obtained: (1) at rest, (2) while the subject held a $15 \mathrm{lb}(6.8 \mathrm{~kg})$ weight isometrically and, (3) during a maximal voluntary isometric contraction. Myotonometer force-displacement curves (amount of tissue displacement to a given unit of force applied perpendicular to the muscle) were compared with sEMG measurements using Pearson's product-moment correlation coefficients. Results: Myotonometer and sEMG measurement correlations ranged from -0.70 to -0.90 . The strongest correlations to sEMG were from Myotonometer force measurements between 1.00 and $2.00 \mathrm{~kg}$. Conclusions: Myotonometer and sEMG measurements were highly correlated. Tissue stiffness, as measured by the Myotonometer, appears capable of assessing changes in muscle activation levels.
\end{abstract}

(C) 2004 Elsevier Ltd. All rights reserved.

Keywords: Electromyography; Myotonometer; Muscle strength; Stiffness; Muscle testing; Rehabilitation

\section{Introduction}

Surface electromyography (sEMG) is a reliable and valid measure of muscle activity, relating directly to changes in torque production during isometric muscle activations $[15,16]$. sEMG, however, is time and laborintensive and data interpretations can be difficult. Time and technical considerations have limited the use of sEMG. The methodology is not widely used in clinical settings, multi-center research studies and large patient population research studies.

The Myotonometer is a computerized, electronic tissue compliance meter that is capable of non-invasive assessment of muscle stiffness at rest and during muscle contraction. Muscle stiffness is typically defined as the

\footnotetext{
${ }^{*}$ Corresponding author. Tel.: +1-406-243-2710; fax: +1-406-2432795.

E-mail address: charles.leonard@umontana.edu (C.T. Leonard).
}

ratio of change in force to change in length along the long axis of a muscle. The change in stiffness to forces applied along the long axis of a muscle is proportional to changes in stiffness to forces applied perpendicular to muscle $[9,17,22]$. The Myotonometer measures stiffness by quantifying resistance (measured as millimeter of tissue displacement) per unit of a perpendicularly applied force. Muscle stiffness measurements obtained during muscle contraction provide an indirect but valid measure of muscle strength $[2,3,7]$. This is possible because a muscle fiber becomes stiffer when stimulated. Muscle stiffness increases linearly with force of contraction and with the same time course as muscle tension $[9,11,22]$. These results have been consistent across various experimental conditions including isolated muscle preparations [10], and during in vivo isometric [3,7], eccentric [17], and isokinetic [3] contractions.

Previous studies have reported on the validity of using compliance meters and the Myotonometer to 
assess tone (resistance to passive stretch while the individual maintains a relaxed state [21]) and strength differences of non-disabled individuals [5,14], individuals with upper motor neuron involvement [14], chronic pain patients [20], and following various exercise or therapeutic interventions $[4,8,17]$.

Myotonometer use has several advantages when compared to sEMG, isokinetic testing and hand-held dynamometry. sEMG setup is time consuming and data interpretations can be difficult. Isokinetic and dynamometry testing can be influenced by muscle substitutions and only measure joint torque, not individual muscle contributions to joint torque. Myotonometer setup time is minimal and data can be acquired faster than other computerized testing procedures. Data acquisition and analysis procedures do not involve extensive user training. Further, intra- and inter-rater reliabilities are very high $[1,13]$.

Previous studies have reported a correlation between muscle stiffness and isokinetic measurements of joint torque $[2,7,9]$. To our knowledge, studies have not examined whether or not a relationship exists between muscle stiffness as assessed by the Myotonometer and muscle activation as measured by sEMG. The purpose of this study was to assess the strength of the correlation between myotonometric measurements and sEMG during various levels of voluntary, isometric muscle contractions of the biceps brachii muscle. Some of the results reported here were published previously in abstract form [12].

\section{Methods}

\subsection{Subjects}

Eight healthy adult subjects (four female; four male), with a mean age of $30.6 \pm 8.23 \mathrm{SD}$ years (range= 22-41 years) were recruited from a sample of convenience of college students. The subjects were screened for orthopedic, pain-related, or other disorders that might influence muscle performance. An injury to either arm within six months prior to the start of the study was an exclusion criteria.

\subsection{Procedures}

All procedures received prior approval from The University of Montana Institutional Review Board for Use of Human Subjects and were conducted in accordance with The Declaration of Helsinki.

The right (dominant) biceps brachii muscle of each subject was tested. Each subject was tested while seated in a chair. The right arm was placed on a platform that was adjustable in height and padded for comfort. The elbow was held in a flexed position at $90^{\circ}$ as determined by a standard hand-held goniometer. The distance between the anterior axillary fold and the center of the antecubital fossa was measured and the midpoint determined. Two marks were placed on the skin - one at a point $2-\mathrm{cm}$ proximal and the other $1-\mathrm{cm}$ proximal to the midpoint. An sEMG electrode $(6 \mathrm{~cm}$, rectangular, bipolar, $\mathrm{Ag}-\mathrm{AgCl}$ with onsite preamplifiers; $2 \mathrm{~cm}$ between active sites) was placed on the skin (following preparation that included shaving, cleansing with alcohol and mild skin abrasion) with its inferior border parallel to the upper mark. A reference electrode was placed on the antero-medial aspect of the left wrist. Recordings at a sampling rate of $2 \mathrm{kHz}$ were amplified $(2-5 \mathrm{~K})$ and high pass filtered at $20 \mathrm{~Hz}$ (Therapeutics Unlimited multichannel EMG amplifier/filtration array; input impedance $15 \mathrm{M} \Omega$ at $100 \mathrm{~Hz}$, noise $<2.0$ microvolt RMS, common mode rejection $87 \mathrm{db}$ at $60 \mathrm{~Hz})$.

The Myotonometer (Neurogenic Technologies, Inc.) is a computerized, electronic device that quantifies tissue stiffness by measuring the amount of resistance encountered as a probe is pushed downward onto the muscle and underlying tissue. The amount of applied pressure (force) is recorded concomitant with the amount of tissue displacement $( \pm 0.1 \mathrm{~mm})$ caused by the pressure of the probe. As increasing probe pressure was applied, tissue displacement measurements were automatically obtained at eight force increments of probe pressure $(0.25,0.50,0.75,1.00,1.25,1.50,1.75$, $2.00 \mathrm{~kg}$ ). Computational software generated force-displacement curves (millimeter tissue displacement per kilogram of probe force) from these data. The higher the resistance (stiffness), the less penetration of the probe and the less steep the slope of the force-displacement curve (Fig. 1).

The head of the Myotonometer probe was placed over the biceps brachii muscle on the distal mark, $1 \mathrm{~cm}$ below and centered along the distal edge of the sEMG electrode. Myotonometer data recordings of all eight force increments take approximately $1 \mathrm{~s}$. Electromyographic data recording length, therefore, was set at $1 \mathrm{~s}$ to correspond to the time required for Myotonometer data acquisition. Myotonometer measurements do not interfere or influence sEMG recordings in any way. Fig. 2 shows the raw sEMG recording during a myotonometric measurement of the biceps brachii at rest. One examiner was responsible for sEMG data collection and another for Myotonometer data acquisition. Each examiner was blinded to the other's data collection.

Three sets of sEMG and Myotonometric data were obtained in the following order: (1) while subjects were at rest, (2) during an isometric contraction while holding a 15-lb (6.8 kg) barbell, and (3) during a maximal voluntary isometric contraction (MVC). The MVC condition involved having subjects maximally contract against a fixed steel cable. A weight was used instead of 

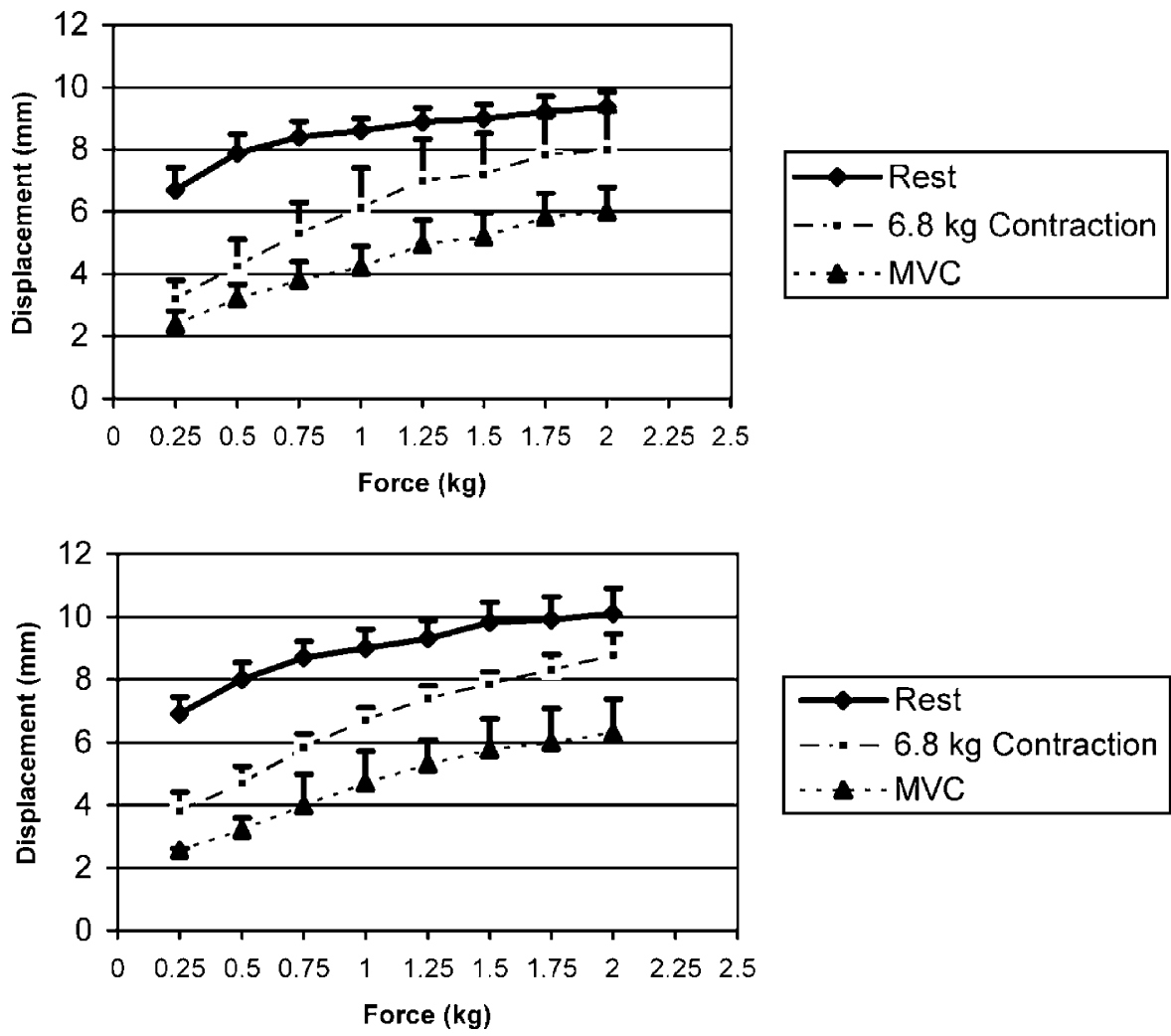

Fig. 1. Myotonometer muscle stiffness measurements (tissue displacement per unit of applied force) of grouped female and male data. Rest: measurements at rest; $6.8 \mathrm{~kg}$ contraction: measurements while subjects held a weight; MVC: maximal voluntary contraction. Note that the stiffness increases with increases in muscle activation. Error bars indicate standard deviations.

a set MVC percentage because this method provided a wider range of sEMG root mean square (RMS) values for correlation analysis. The elbow was positioned in $90^{\circ}$ flexion, the wrist and shoulder in anatomical neutral, for MVC and barbell trials. Eight trials were performed for each of the three testing conditions. The

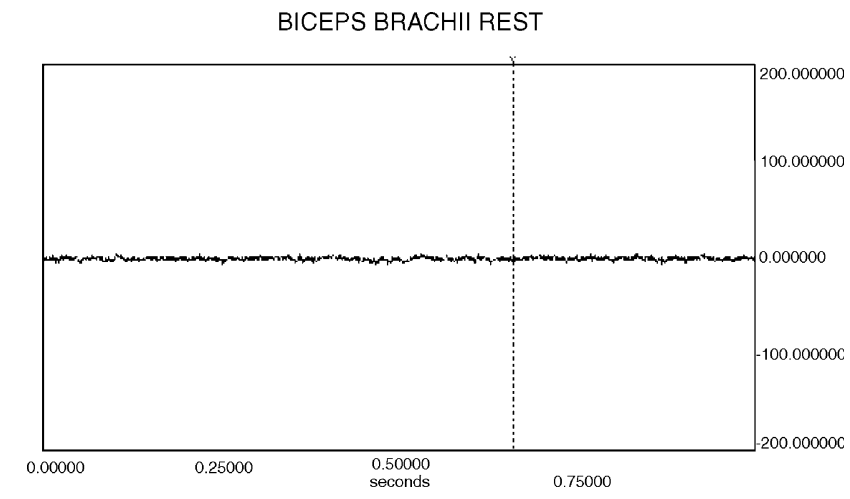

Fig. 2. A computer screen printout of one, 1-s trial with the biceps brachii at rest. The vertical dotted line corresponds to an audible tone emitted by the Myotonometer signaling a $2.0 \mathrm{~kg}$ probe force pressure has been obtained and the myotonometric measurement for the trial has been completed. Myotonometric measurements had no effect on sEMG recordings. subjects were verbally cued to begin contraction prior to the start of EMG and Myotonometer data collection and were instructed to maintain the contraction for $1 \mathrm{~s}$ post data collection. Sufficient rest was given between each trial to help eliminate the effects of fatigue.

Surface EMG values were expressed in terms of the RMS of the amplitude for each 1-s trial. Myotonometer values were expressed in terms of millimeters of displacement per kilogram of applied force to the muscle. Mean values for the eight trials of each subject for each testing condition were calculated.

\subsection{Statistical analysis}

Myotonometer computational software calculated the area under the curve (AUC) for each force-displacement curve. The degree of relationship between biceps brachii muscle RMS and myotonometric measurements was determined using a Pearson's productmoment correlation coefficient. Correlations between RMS and myotonometric measurements were calculated for all the eight subjects combined and with respect to gender. Correlations were calculated for the entire slope of female and male subjects' force-dis- 
placement curves and for each of the eight incremental Myotonometer force measurements $(0.25-2.00 \mathrm{~kg})$. These analyses permitted the determination of the relationship between the amount of tissue displacement to a perpendicularly applied force (stiffness) with changing sEMG levels for individual force increments (i.e. the level of correlation of each perpendicularly applied force increment with sEMG). Additionally, the strength of the relationship between the AUC of the slope generated by all eight force increment measurements (Fig. 1) and corresponding change in sEMG was determined. Probability values of less than 0.05 were considered significant.

\section{Results}

\subsection{Female and male RMS and Myotonometer data correlations}

Each Myotonometer measurement resulted in a force-displacement curve that reflected the amount of tissue displacement per unit force (Fig. 1). The AUC of these force-displacement curves was calculated for each subject for each condition (rest, $6.8 \mathrm{~kg}$, MVC). Female and male grouped AUC data were correlated to RMS data. The mean MVC for males was $967.15 \pm 368.41$ microvolt $\quad$ (range $=837.78-1471.25$ microvolt). The mean MVC for females was $436.93 \pm 75.42$ microvolt (range $=353.38-559.25$ microvolt). The correlation between AUC and RMS data for females was -0.84 $(p=0.001)$. The correlation between AUC and RMS data for males was $-0.85(p=0.000)$. Fig. 1 shows grouped female and male muscle stiffness values for each of the three muscle contraction conditions.

\subsection{RMS and individual Myotonometer force data correlations}

Table 1 summarizes results obtained from individual Myotonometer force measurements of female and male subjects. Correlation coefficients ranged from -0.70 to -0.86 for female subjects. Correlations for male subjects ranged from -0.71 to -0.90 . Significance was obtained for five of the eight Myotonometer force measurements. These five measurements represented the higher Myotonometer force levels $(1.00-2.00 \mathrm{~kg})$.

\subsection{Combined group: RMS and Myotonometer data correlations}

Table 2 summarizes results obtained for all the eight subjects. Correlations among RMS and Myotonometer measurements ranged from -0.57 to -0.70 . Significant relationships were documented for seven of the eight Myotonometer force measurements $(0.25$ and 0.75 to $2.00 \mathrm{~kg})$.
Table 1

Correlation coefficients between sEMG-RMS and Myotonometer measurements

\begin{tabular}{lll}
\hline $\begin{array}{l}\text { Myotonometer } \\
\text { force }(\mathrm{kg})\end{array}$ & $\begin{array}{l}\text { Correlation EMG- } \\
\text { Myotonometer }(r)\end{array}$ & Probability $(p)$ \\
\hline $\begin{array}{l}\text { Female subjects } \\
0.25\end{array}$ & -0.70 & \\
0.50 & -0.76 & 0.42 \\
0.75 & -0.81 & 0.15 \\
1.00 & -0.84 & 0.06 \\
1.25 & -0.85 & $\mathbf{0 . 0 3}$ \\
1.50 & -0.86 & $\mathbf{0 . 0 2}$ \\
1.75 & -0.86 & $\mathbf{0 . 0 1}$ \\
2.00 & -0.86 & $\mathbf{0 . 0 1}$ \\
& & $\mathbf{0 . 0 1}$ \\
Male subjects & & \\
0.25 & -0.71 & \\
0.50 & -0.75 & 0.34 \\
0.75 & -0.79 & 0.17 \\
1.00 & -0.84 & 0.08 \\
1.25 & -0.88 & $\mathbf{0 . 0 2}$ \\
1.50 & -0.89 & $\mathbf{0 . 0 0}$ \\
1.75 & -0.90 & $\mathbf{0 . 0 0}$ \\
2.00 & -0.89 & $\mathbf{0 . 0 0}$ \\
\hline
\end{tabular}

Table 2

Correlation coefficients between sEMG-RMS and Myotonometer measurements

\begin{tabular}{lcc}
\hline $\begin{array}{l}\text { Myotonometer } \\
\text { force }(\mathrm{kg})\end{array}$ & $\begin{array}{l}\text { Correlation EMG- } \\
\text { Myotonometer }(r)\end{array}$ & Probability $(p)$ \\
\hline $\begin{array}{l}\text { Combined group (female } \\
0.25\end{array}$ & -0.57 \\
0.50 & -0.60 & $\mathbf{0 . 0 1}$ \\
0.75 & -0.64 & 0.06 \\
1.00 & -0.67 & $\mathbf{0 . 0 3}$ \\
1.25 & -0.70 & $\mathbf{0 . 0 1}$ \\
1.50 & -0.70 & $\mathbf{0 . 0 1}$ \\
1.75 & -0.70 & $\mathbf{0 . 0 1}$ \\
2.00 & -0.67 & $\mathbf{0 . 0 1}$ \\
\hline
\end{tabular}

\section{Discussion}

Results documented high to very high correlations among Myotonometer and RMS measurements when data were compared with respect to gender. The highest and most significant correlations among Myotonometer measurements and sEMG recordings occurred at the higher increments of Myotonometer application force. This is best explained by the fact that the Myotonometer probe must displace a certain amount of cutaneous and subcutaneous tissue before it begins to displace the targeted muscle tissue. Myotonometer measurements during the lower force recordings reflect, in part, displacement of non-contractile tissue and thus a weaker correlation to sEMG recordings when compared with measurements obtained at higher Myotonometer force recordings. 
Correlation values between sEMG and myotonometric measurements for the combined group would be considered in the moderate to good ranges. That combined group correlations were lower than when data were compared with respect to gender can be explained by an aggregation effect [19], explained as follows. Men and women possess inherent anatomical and physiological differences in subcutaneous tissues that can influence results when data are combined. The difference in RMS values for the MVC condition between females and males was very high (Fig. 3). When males and females were combined for statistical purposes, the high variance in the data decreased the strength of the correlation. When data for the female and male groups were separately analyzed, the variance decreased. Gender-specific correlation values represent a truer physiological picture.

A potential benefit of Myotonometer use in clinical settings is its ability to quantify changes in muscle activations quickly and easily. This is an improvement over manual muscle testing and other subjective assessment tools that only provide qualitative assessments of these changes. In addition, there are problems with manual muscle testing reliabilities especially in strength ranges above the fair range $[6,18]$ and measurements are not sensitive enough to assess small or moderate changes in muscle strength [18]. Very high intra- and inter-rater reliabilities for both children and adults have been reported for myotonometric measurements $[1,13]$ and measurements are capable of detecting small changes [14]. Unlike measurements by computerized or hand-held dynamometers, Myotonometer measurements are not compromised by the possibility of muscle substitutions and can assess individual muscles and not just total joint torques. Because the Myotonometer is capable of assessing muscle tone changes at rest, it might be helpful in cases where joint movement or contraction causes pain. The Myotonometer is not influenced by atmospheric pressure changes and might be useful in assessing muscle health in microgravity environments. A considerable advantage is that Myotonometer setup and data acquisition and analysis requires

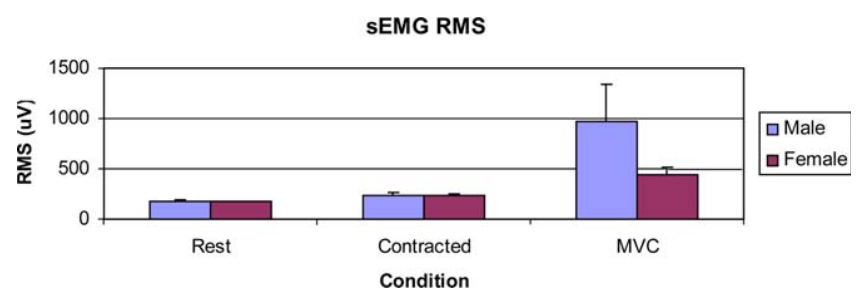

Fig. 3. Surface electromyography (sEMG) root mean square (RMS) data for female and male subjects. Rest: RMS of biceps brachii at rest; contracted: RMS of subjects while holding a $6.8 \mathrm{~kg}$ weight; MVC: RMS during a maximal voluntary contraction. Error bars indicate standard deviations. considerably less time than sEMG or other computerized testing. During testing procedures used for this study, eight trials of eight separate force measurements could be acquired in approximately $10 \mathrm{~s}$.

Prior studies that have demonstrated the relationship between muscle stiffness and activation levels have used longitudinally applied torques to the muscle to measure stiffness. The present study is the first that we are aware of to demonstrate that a similar relationship exists between stiffness measurements obtained by a perpendicularly applied force to the muscle and muscle activation levels. Myotonometer operation and technical requirements are considerably easier than those of the various apparatuses that have been used to measure stiffness by applying a force longitudinal to the muscle.

The present study was conducted using a sample of healthy adult subjects. Pathological changes of muscle associated with various disabilities might demonstrate different correlation values. Further studies are needed to establish the effectiveness of the Myotonometer in assessing changes in a muscle's activation and torque generating ability in patient populations.

\section{Conclusions}

Myotonometer measurements of biceps brachii muscle stiffness are highly correlated to sEMG changes in healthy adult subjects. Myotonometer force-displacement measurements are sensitive to changes in muscle activation levels. The results of this study indicate that the Myotonometer can be used as an objective means to non-invasively and quickly assess changes in resting muscle stiffness and muscle activation.

\section{Acknowledgements}

The authors would like to express thanks to Carl Heine, Ph.D., and Daniel Sandholdt for technical assistance and to Kathleen Miller, Ph.D., for statistical analysis assistance. This work was made possible by funding provided from the MJ Murdock Charitable Trust Foundation.

\section{References}

[1] D. Aarrestad, M. Williams, S. Fehrer, E. Mikhailenok, C. Leonard, Intra- and inter-rater reliabilities of the Myotonometer when assessing the spastic condition of children with cerebral palsy, Child Neurol., in press.

[2] M. Bizzini, A. Mannion, Reliability of a new, hand-held device for assessing skeletal muscle stiffness, Clin. Biomech. 18 (2003) $459-461$. 
[3] S. Cannon, G. Zahalak, The mechanical behavior of active human skeletal muscle in small oscillations, J. Biomech. 15 (1982) 111-121.

[4] T. Coon, E. Ikeda, J. Lamb, D. Sebastian, The effects of straincounterstrain on muscle hardness and tenderness in subjects with neck pain, J. Orthop. Sports Phys. Ther. 32 (2002) A29.

[5] A.A. Fischer, Muscle tone in normal persons measured by tissue compliance, J. Neurol. Orthop. Med. Surg. 8 (1987) 227-233.

[6] E. Frese, M. Brown, B.J. Norton, Clinical reliability of manual muscle testing. Middle trapezius and gluteus medius muscles, Phys. Ther. 67 (7) (1987) 1072-1076.

[7] G.I. Gevlich, L.S. Grigoryeva, M.I. Boyko, I.B. Kozlovskaya, Evaluation of skeletal muscle tone by recording lateral rigidity, Kosm. Biol. Aviakosm. Med. 17 (1983) 86-89.

[8] M. Horikawa, Effect of visual display terminal height on the trapezius muscle hardness: quantitative evaluation by a newly developed muscle hardness meter, Appl. Ergonomics 32 (2001) 473-478.

[9] M. Horikawa, S. Ebihara, F. Sakai, M. Akiyama, Non-invasive measurement method for hardness in muscular tissues, Med. Biol. Eng. Comput. 31 (1993) 623-627.

[10] G.C. Joyce, P.M.H. Rack, Isotonic shortening and lengthening movements of cat soleus muscle, J. Physiol. 204 (1969) 475-491.

[11] N. Lan, P. Crago, Optimal control of antagonistic muscle stiffness during voluntary movements, Biol. Cybern. 71 (1994) 123135 .

[12] C. Leonard, J. Brown, T. Price, S. Queen, E. Mikhailenok, Comparison of surface electromyography and myotonometric measurements during isometric contractions, Arch. Phys. Med. Rehabil. 83 (2002) 1683.

[13] C. Leonard, W. Deshner, J. Romo, E. Suoja, S. Fehrer, E. Mikhailenok, Myotonometer intra- and inter-rater reliabilities, Arch. Phys. Med. Rehabil. 84 (2003) 928-932.

[14] C. Leonard, J. Stephens, S. Stroppel, Assessing the spastic condition of individuals with upper motoneuron involvement: validity of the Myotonometer, Arch. Phys. Med. Rehabil. 82 (2001) $1416-1420$.

[15] W.N. Loscher, A.G. Cresswell, A. Thorstensson, Electromyographic responses of the human triceps surae and force tremor during sustained submaximal isometric plantar flexion, Acta Physiol. Scand. 152 (1994) 73-82.

[16] T. Moritani, Y. Yoshitake, The use of electromyography in applied physiology, J. Electromyogr. Kinesiol. 8 (1998) 363-381.

[17] M. Murayama, K. Nosaka, T. Yoneda, K. Minamitani, Changes in hardness of the human elbow flexor muscles after eccentric exercise, Eur. J. Appl. Physiol. 82 (2000) 361-367.

[18] L. Noreau, J. Vachon, Comparison of three methods to assess muscular strength in individuals with spinal cord injury, Spinal Cord 36 (1998) 716-723.

[19] L. Portney, M. Watkins, Foundations of Clinical Research: Applications to Practice, Appleton and Lange, Connecticut, 1993.

[20] F. Sakai, S. Ebihara, M. Akiyama, M. Horikawa, Pericranial muscle hardness in tension-type headache: a non-invasive measurement method and its clinical application, Brain 118 (1995) 523-531.

[21] T. Sanger, M.R. Delgado, D. Gaebler-Spira, et al., Classification and definition of disorders causing hypertonia in childhood, Pediatrics 111 (2003) 89-97.

[22] E. Walsh, Muscles, masses and motion, The Physiology of Normality, Hypotonicity, Spasticity and Rigidity, MacKeith Press, London, 1992.

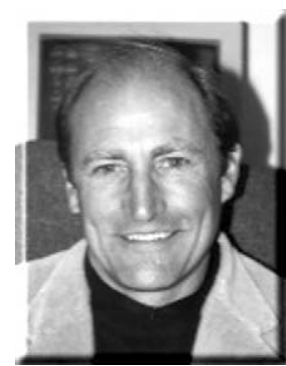

Dr. Leonard received a BS degree from the University of Delaware, an MS in Physical Therapy from the Duke University and a Ph.D. in Neuroscience from the Medical College of Pennsylvania. He was an $\mathrm{NIH}$ postdoctoral fellow at the Nobel Institute of Neurophysiology and Department of Pediatrics, Karolinska Institute. He is currently a Professor and Director of the Motor Control Research Laboratory at the University of Montana. He is the co-patent holder of The Myotonometer. He is one of the founding members and President of Neurogenic Technologies, Inc.

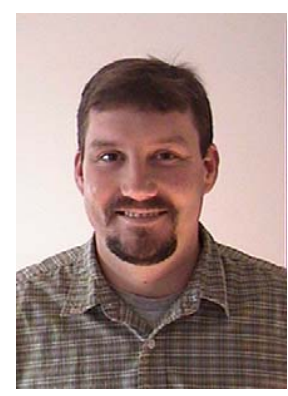

Jason graduated cum laude from the University of Montana with a BS degree in Physical Therapy. He currently practices as an outpatient clinician and is raising his fine family in Duluth, Minnesota.

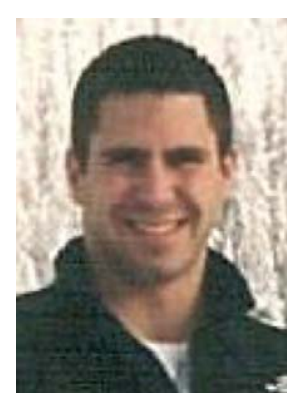

Tim graduated from the University of Montana with a BS degree in Physical Therapy. $\mathrm{He}$ is currently the Rehabilitation Coordinator for Evergreen Health and Rehabilitation in Polson, Montana.

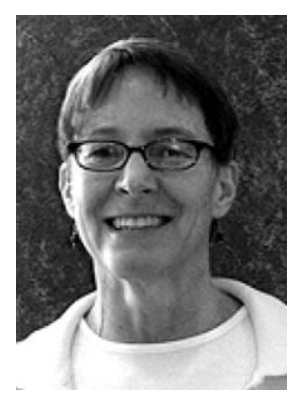

Dr. Queen received a BS in Physical Therapy from the University of Colorado and a $\mathrm{Ph} . \mathrm{D}$. in Medical Sciences from the University of New Mexico. At the University of Kentucky, she is an Assistant Professor in the Division of Physical Therapy, Department of Rehabilitation Sciences and a faculty associate of the Spinal Cord and Brain Injury Research Center.

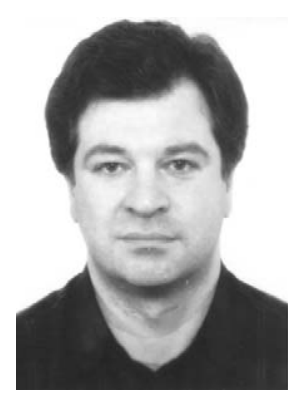

Dr. Mikhailenok received his MD from the Leningrad Pediatric Medical Institute and his Ph.D. in Human Physiology from the Institute for Experimental Medicine, Russian Academy of Medical Sciences. He is the copatent holder of The Myotonometer and is the Vice-President of Research and Development for Neurogenic Technologies, Inc. 